\title{
Addendum: Chaos around a Hénon-Heiles-Inspired Exact Perturbation of a Black Hole
}

\author{
Werner M. Vieira* and Patricio S. Letelier ${ }^{\dagger}$ \\ Departamento de Matemática Aplicada \\ Instituto de Matemática, Estatística e Ciências da Computação \\ Universidade Estadual de Campinas, CP 6065 \\ 13081-970 Campinas, SP, Brazil
}

(August 29, 1996)

\begin{abstract}
We find that the model of a black hole plus an exterior halo of quadrupoles and octopoles recently proposed by us is more chaotic than previously detected. In fact, the quadrupolar component gives rise also to a chaotic behavior, found after further numerical search. This fact reinforces even more the role of chaos in relativistic core-halo models.
\end{abstract}

PACS numbers: 04.20.Jb, 05.45.+b, 95.10.Fh, 95.30.Sf

Typeset using REVTEX

*e-mail: vieira@ime.unicamp.br

†e-mail: letelier@ime.unicamp.br 
We have recently proposed [1] an exact relativistic model to describe core-halo type gravitational systems. The astronomical motivation for the model was a massive black hole or a galactic nucleus at the center, surrounded by an also massive, exterior halo of dust. This halo does not need to be planar and was inspired by the classical Hénon-Heiles system in the Newtonian limit of General Relativity. To this end, we retained both the quadrupolar and octopolar terms in the halo expansion. The core-halo solution was constructed in Weyl metric, where a kind of nonlinear superposition of distinct solutions is known to be possible.

We also studied in [1] the influence of the exterior halo on the geodesic motion in the inner vacuum. We detected the occurrence of large chaotic regions due to the presence of the octopolar halo component (see Fig. 1(b) of that Reference) and an apparent preservation of integrability when only the quadrupolar halo component was taken into account (see Fig. 1(c) there).

In this note we report the occurrence of chaos even when we have a purely quadrupolar halo. In this case, the chaotic regions are much smaller than in the octopolar case, being found after a more detailed numerical search. This is shown in Fig. 1, which is to be compared with Fig. 1(c) of [1]. It stresses the fact that chaos is more conspicuous in our model than previously detected and hence reinforces its role in relativistic core-halo models. On the other hand, this additional numerical finding does not alter any other conclusion of [1]; in particular, the presence of both the quadrupolar and the octopolar terms are indispensable to accomplish the Hénon-Heiles-like form of the halo in the Newtonian limit.

The additional finding above recalls us of a basic question: how much information is available about a system from Poincaré's sections? Although powerful to identify in an invariant way chaotic signatures in phase space, we know that it is a numeric tool and hence it is insufficient in any case to assure full integrability.

In [2] a perturbation of the Schwarzschild background due to an exterior halo of matter is also considered. It reduces in the Newtonian limit to the quadrupolar gravitational moment (instead of a dipolar one as found in [1]). In [3] such a multipolar expansions are also considered for Majumdar-Papapetrou solutions. In constrast with this, each one of our 
$n^{\text {th }}$-pole, besides being per se an exact solution, it is a pure one in the sense that it leads to the corresponding Newtonian pole in that limit. Finally, Moeckel's claim that his model is the relativistic analog of the famous Hill problem in celestial mechanics is unclear and will be considered in another work.

The authors thank CNPq and FAPESP for financial support. 


\section{REFERENCES}

[1] Vieira, W. M. and Letelier, P. S., Phys. Rev. Lett. 76, 1409 (1996). See also Errata in Phys. Rev. Lett. 76, 4098 (1996).

[2] R. Moeckel, Comm. Math. Phys. 150, 415 (1992).

[3] Cornish, N. J. and Frankel, N. E., University of Melbourne Report UM-P-96/45 (1996). See also list gr-qc/9606021. 


\section{FIGURES}

FIG. 1. This figure is in all respects the same as Fig. 1(c) of [1], except that it contains much more points, this time sufficient to exhibit (three small) chaotic regions (one around each vertex of the triangular region). 


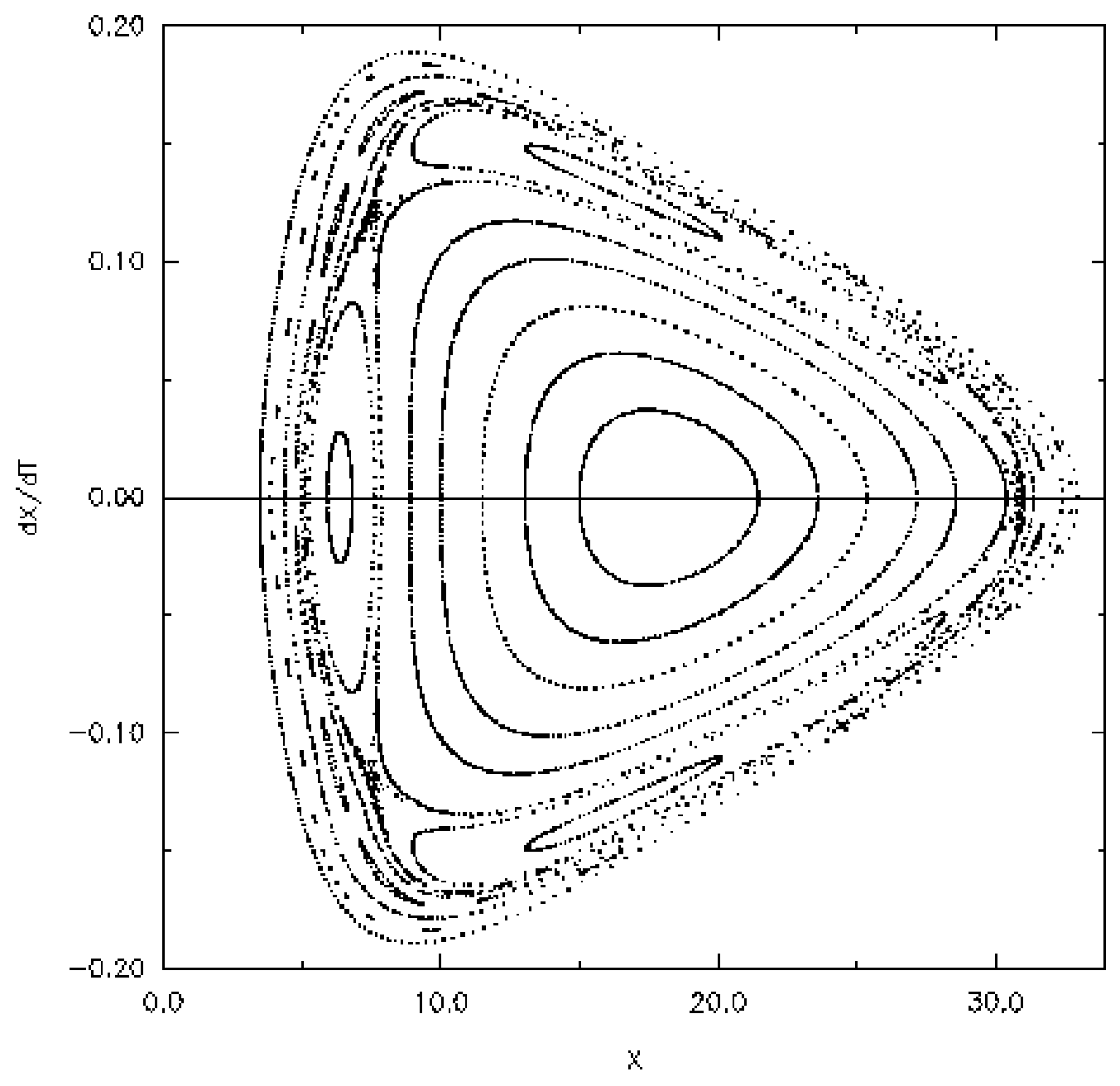

